\title{
Continuation of Bicharacteristics for Effectively Hyperbolic Operators ${ }^{\dagger}$
}

\author{
By \\ Gen Komatsu* and Tatsuo Nishitani**
}

Introduction

The present paper is concerned with bicharacteristics of an effectively hyperbolic differential or pseudo-differential operator. Given a hyperbolic principal symbol $p=p(\rho)=p(x, \xi)$ and an effectively hyperbolic characteristic point $\hat{\rho}=(\hat{x}, \hat{\xi}) \in T^{*} R^{n+1}$, we consider bicharacteristics $\rho=\rho(s)$ tending to $\hat{\rho}$ as $s \uparrow+\infty$ or $s \downarrow-\infty$. Our main purpose is to prove the following two facts (cf. Theorem 1):

(0.1) There are exactly four such bicharacteristics. Two of them are incoming toward the reference point $\hat{\rho}$ with respect to the parameter $s$, and the other two are outgoing.

(0.2) Each one of the incoming (resp. outgoing) bicharacteristics is naturally continued to the other one, and the resulting two curves are regular, $C^{\infty}$ or analytic corresponding to the assumption on the principal symbol.

Concerning (0.1), it was first observed by Melrose [12] that there are at least four such bicharacteristics. Following that work, the second named author showed in [13] that the number of such bicharacteristics is exactly four, under a smallness assumption on the purely imaginary eigenvalues of the Hamilton map. This assumption was then eliminated by Iwasaki [11] by using his result in [10] concerning a factorization of the principal symbol. He also observed the $C^{\infty}$ result in $(0.2)$, the $C^{\infty}$ continuation of

Communicated by S. Matsuura, March 7, 1989.

1991 Mathematics Subject Classification: 35L 10

+ This work was supported by Grant-in-Aid for Scientific Research, The Ministry of Education, Science and Culture.

* Department of Mathematics, Osaka University, Toyonaka 560, Japan

** Department of Mathematics, College of General Education, Osaka University, Toyonaka 560, Japan 
bicharacteristics. The method employed in [10] is the Nash-Moser implicit function theorem or, rather, its proof. It will be extremely difficult, even if it is possible, to obtain an analytic version of the result in [10].

Our method of proving (0.1) and (0.2) above is completely elementary and applies equally to the analytic case as well as the $C^{\infty}$ case; rather, the analytic case is much simpler. We reduce the problem to the study of a Briot-Bouquet singularity (cf. Theorem 2), where a linear algebraic condition appears quite naturally. Nevertheless, it is plausible that the same idea is applied also to a higher order analogue of effectively hyperbolic principal symbol. We hope to return to this matter at a future time.

It has been known that the Cauchy problem for a hyperbolic operator is $C^{\infty}$ well-posed independent of the choice of lower order terms if and only if the principal symbol is effectively hyperbolic at every multiple (necessarily double) characteristic point. (There were many contributions of proving this fact in general, and Iwasaki's factorization theorem in [10] was fundamental in order to reduce the general problem to a special case.) The notion of effective hyperbolicity was first introduced by Ivrii and Petkov in [9]. Its definition requires the existence of (necessarily two) non-vanishing real eigenvalues of the Hamilton map i.e. the so-called fundamental matrix obtained by linearizing the Hamilton field of the principal symbol. (It turns out that such eigenvalues must be of the form $\pm \lambda$.) The question arises how this linear algebraic definition is reflected in the dynamical system of bicharacteristics near the reference point. This is a motivation of the present work.

The present paper is organized as follows. The main result (Theorem 1) is stated in Subsection 1.1. We then exhibit, in Subsection 1.2, a heuristic argument by considering a linearized problem. An outline of the proof of Theorem 1 is given in Subsection 1.3, where we reduce the problem to the study of a singular initial value problem (cf. Theorem 2). This reduction involves a symplectic change of coordinates, which we achieve in Subsection 2.1. Using the new coordinates $\rho=(y, \eta)$ with $\hat{\rho}=(0,0)$, we get a factorization of the form $p=p^{+} p^{-}$with

$$
p^{ \pm}(y, \eta)=\eta_{0}-y_{0} \pi^{ \pm}\left(y_{0}, v / y_{0}\right), v=\left(y^{\prime}, \eta^{\prime}\right),
$$

cf. (1.7), a factorization which is only valid within a restricted neighborhood of $\hat{\rho}$. This restriction causes no difficulty (cf. Proposition 1), as we see in Subsection 2.2. Thus, Section 2 concerns the reduction of the proof of 
Theorem 1 to that of Theorem 2. We prove Theorem 2 in Section 3 by using (the $C^{\infty}$ version of) the Briot-Bouquet theorem due to Briot-Bouquet [1] (and de Hoog-Weiss [3], [4] for the $C^{\infty}$ version, see also Russell [14]). We first state, in Subsection 3.1, the original holomorphic Briot-Bouquet theorem in system form (Proposition 2). This implies Theorem 2 in the analytic case, whereas the $C^{\infty}$ version (Proposition 3), formulated as a one-sided problem, reduces the proof of Theorem 2 to examining the consistency of the successive right and left derivatives of the solution at the origin. This step requires some knowledge of a related linear system, which is also used in the proof of Proposition 3-we prepare it in Subsection 3.2. Finally in Subsection 3.3, we complete the proof of Theorem 2 after stating and proving Proposition 3.

\section{$\S 1$. Statement of the Results and Outline of the Proof}

\subsection{Continuation for two pairs of bicharacteristics}

Suppose given a linear partial differential (or pseudo-differential) operator $P=P\left(x, D_{x}\right), D_{x}=-\sqrt{-1} \partial / \partial x$, of order $m$ with $C^{\infty}$ coefficients in an open set $\Omega \subset \boldsymbol{R}^{n+1}$ with coordinates $x=\left(x_{0}, x^{\prime}\right)=\left(x_{0}, x_{1}, \cdots, x_{n}\right)$. We denote by $p=p(\rho)=p(x, \xi)$ the (real) principal symbol of $P$, so that $p \in C^{\infty}\left(T^{*} \Omega \backslash 0\right)$, where the cotangent bundle $T^{*} \Omega$ is equipped with the standard coordinates $\rho=$ $(x, \xi), \xi=\left(\xi_{0}, \xi^{\prime}\right)=\left(\xi_{0}, \xi_{1}, \cdots, \xi_{n}\right)$. Suppose also given a multiple characteristic reference point $\hat{\rho}=(\hat{\mathrm{x}}, \hat{\xi}) \in \Sigma_{2}=\Sigma \backslash \Sigma_{1}$, where

$$
\Sigma=\left\{\rho \in T^{*} \Omega \backslash 0 ; p(\rho)=0\right\}, \quad \Sigma_{1}=\{\rho \in \Sigma ; d p(\rho) \neq 0\},
$$

so that $\Sigma_{1}$ is the simple characteristic set of $p$. We assume that $p(x, \cdot)$ is hyperbolic with respect to $d x_{0}$ near $x=\hat{x} \in \Omega$, that is, the equation $p(x, \xi)=0$ $(\xi \neq 0)$ with unknown $\xi_{0}$ has exactly $m$ real zeros $\xi_{0}=\xi_{0}\left(x, \xi^{\prime}\right)$ near $x=\hat{x}$ counted with multiplicity.

Setting $(y, \eta)=(x-\hat{x}, \xi-\xi) \in \boldsymbol{R}^{2 n+2}$, we obtain a new coordinate system $\rho=(y, \eta)$ on $T^{*} \Omega$ for which $\hat{\rho}$ is the origin. It then follows from the Taylor expansion around $\rho=\hat{\rho}$ that

$$
p(\rho)=p_{\hat{\rho}}(y, \eta)+O\left(|(y, \eta)|^{3}\right) \quad \text { as } \quad \rho \rightarrow \hat{\rho},
$$

where $p_{\hat{\rho}}(y, \eta)$ is a quadratic polynomial of $(y, \eta)$ corresponding to the 
Hessian of $\frac{1}{2} p(\rho)$ at $\rho=\hat{\rho}$. Observe that this quadratic polynomial extends naturally to a quadratic form $Q(\cdot, \cdot)$ on $T_{\hat{\rho}}\left(T^{*} \Omega\right)$. Then, the Hamilton map $\mathscr{H}$ of $p$ at $\hat{\rho}$ is defined by

$$
\sigma(X, \mathscr{H} Y)=Q(X, Y) \quad \text { for } \quad X, Y \in \mathbb{T}_{\hat{\rho}}\left(T^{*} \Omega\right),
$$

where $\sigma$ stands for the natural symplectic form given by

$$
\sigma=\sum_{j=0}^{n} d \xi_{j} \wedge d x_{j}=\sum_{j=0}^{n} d \eta_{j} \wedge d y_{j}
$$

Recall that the matrix expression of $\mathscr{H}$ with respect to the coordinates $(y, \eta)$ is the so-called fundamental matrix given by

$$
\mathscr{H}=\frac{1}{2}\left(\begin{array}{cc}
p_{\eta y} & p_{\eta \eta} \\
-p_{y y} & -p_{y \eta}
\end{array}\right) \quad \text { evaluated at } \rho=\hat{\rho}
$$

where the subscripts $y, \eta$ stand for the differentiation.

Let us assume that $p=p(\rho)$ is effectively hyperbolic at $\rho=\hat{\rho}$, that is, the Hamilton map $\mathscr{H}$ admits at least one non-vanishing real eigenvalue. (It turns out that the number of such eigenvalues is exactly two, if there exist, and that they must be of the form $\pm \lambda, 0 \neq \lambda \in \mathbb{R}$.) Clearly, this condition is open with respect to points $\rho \in \Sigma_{2}$. Observe that $\hat{\rho}$ is a double characteristic point, because the quadratic form $Q(\cdot, \cdot)$ does not vanish identically.

We are interested in the behavior of bicharacteristics of $p=p(\rho)$ near $\rho=\hat{\rho}$. By definition, a (null) bicharacteristic of $p$ is an integral curve $\rho=\rho(s)$ of the Hamilton field

$$
H_{p}=\sum_{j=0}^{n}\left(\frac{\partial p}{\partial \xi_{j}} \frac{\partial}{\partial x_{j}}-\frac{\partial p}{\partial x_{j}} \frac{\partial}{\partial \xi_{j}}\right)=\frac{\partial p}{\partial \xi} \cdot \frac{\partial}{\partial x}-\frac{\partial p}{\partial x} \cdot \frac{\partial}{\partial \xi}
$$

subject to the restriction $p(\rho(s))=0$. In terms of the coordinates $\rho=(y, \eta)$, this curve $\rho=\rho(s)$ satisfies the following system of ordinary differential equations

$$
\frac{d y}{d s}(s)=\frac{\partial p}{\partial \eta}(y(s), \eta(s)), \quad \frac{d \eta}{d s}(s)=-\frac{\partial p}{\partial y}(y(s), \eta(s))
$$

the Hamilton system with Hamilton function $p=p(y, \eta)$. It is worthwhile 
noting that the linearization of (1.1) at $\rho=\hat{\rho}$ yields a matrix corresponding to $2 \mathscr{H}$.

We are thus led to study solutions of (1.1) satisfying

$$
(y(s), \eta(s)) \rightarrow(0,0) \quad \text { as } \quad s \uparrow+\infty \quad \text { (resp. } s \downarrow-\infty) \text {; }
$$

we refer to them as incoming (resp. outgoing) bicharacteristics relative to the reference point $\rho=\hat{\rho}$. Two bicharacteristics will be identified if they are equivalent under a trivial change of parameters $s \rightarrow s+$ constant. Now, the main result of the present paper is stated as follows:

Theorem 1. There are exactly two incoming (resp. outgoing) bicharacteristics of $p=p(\rho)$, relative to $\rho=\hat{\rho}$, contained in the simple characteristic set $\Sigma_{1}$. Furthermore, one of the incoming (resp. outgoing) bicharacteristics is naturally continued to the other one, and the resulting two curves are $C^{\infty}$ regular near $\rho=\hat{\rho}$ as submanifolds of $T^{*} \Omega$. These two curves are (real) analytic near $\rho=\hat{\rho}$ whenever $p=p(\rho)$ is assumed to be analytic there.

\subsection{A linearized model}

(A). A linearized problem. Before beginning the proof of Theorem 1 above, we wish to exhibit a heuristic argument. Readers can skip it and proceed directly to the next Subsection, in which the proof of Theorem 1 is outlined.

It may be natural to expect that our problem (1.1) with (1.2) is a perturbation of its linearization at $\rho=\hat{\rho}$ :

$$
\frac{d \tilde{\rho}}{d s}(s)=2 \mathscr{H} \tilde{\rho}(s), \quad \tilde{\rho}=(y, \eta) \in \boldsymbol{R}^{n+1} \times \boldsymbol{R}^{n+1},
$$

under the limit condition (1.2), where $\tilde{\rho}=(y, \eta)$ is regarded as a column vector. To consider $(1.1)^{\prime}$ is equivalent to replacing $p(\rho)$ by its quadratic part $p_{\hat{\rho}}(\tilde{\rho})$. After a linear symplectic change of coordinates, we can write $p_{\hat{\rho}}(\tilde{\rho})=Q(\tilde{\rho}, \tilde{\rho})$ in normal form - up to non-vanishing multiplicative factor,

$$
\begin{aligned}
Q(\tilde{\rho}, \tilde{\rho}) & =\eta_{0}^{2}-y_{0}^{2}-\sum_{j=1}^{n_{1}} \mu_{j}\left(y_{j}^{2}+\eta_{j}^{2}\right)-\mu_{0} \sum_{j=n_{1}+1}^{n_{2}} y_{j}^{2} \\
& =\sum_{j=0}^{n} Q_{j}\left(\tilde{\rho}_{j}, \tilde{\rho}_{j}\right) \quad \text { for } \quad \tilde{\rho}_{j}=\left(y_{j}, \eta_{j}\right) \in \mathbb{R}^{2},
\end{aligned}
$$


where $\mu_{j}, \mu_{0}>0$ and $0 \leq n_{1} \leq n_{2} \leq n$ (cf. (1.3) with (1.5) and the proof of Lemma 2.2 below). Therefore, $(1.1)^{\prime}$ splits into real $2 \times 2$ systems

$(1.1)^{\prime}{ }_{j} \quad \frac{d}{d s} \tilde{\rho}_{j}(s)=2 \mathscr{H}_{j} \tilde{\rho}_{j}(s) \quad$ for $\quad 0 \leq j \leq n$,

where each matrix $\mathscr{H}_{j} \in M_{2}(\mathbb{R})$ corresponds to $Q_{j}\left(\tilde{\rho}_{j}, \tilde{\rho}_{j}\right)$. We are thus led to consider $(1.1)^{\prime}{ }_{j}$ under the condition

$$
\tilde{\rho}_{j}(s) \rightarrow(0,0) \in \mathbb{R}^{2} \quad \text { as } s \downarrow-\infty \quad \text { or } \quad s \uparrow+\infty \text {. }
$$

It is well known in the linear theory of ordinary differential equations that the nature of the equilibrium point $(0,0)$ is determined by the spectral property of the real matrix $\mathscr{H}_{j}$ : if $j=0$ (resp. $1 \leq j \leq n_{1}$ ), then $\mathscr{H}_{j}$ has eigenvalues \pm 1 (resp. $\pm \sqrt{-1} \mu_{j}$ ), so that the origin $(0,0)$ is a saddle point (resp. center); if $n_{1}<j \leq n_{2}$ (resp. $\left.n_{2}<j \leq n\right)$, then $\operatorname{rank} \mathscr{H}_{j}=1\left(\operatorname{resp} . \mathscr{H}_{j}=0\right)$ and $\mathscr{H}_{j}$ has only one eigenvalue 0 . Therefore,

Claim. If $1 \leq j \leq n$ then there are no (non-trivial) singular trajectories, that is, $\tilde{\rho}_{j}(s)=(0,0)$ is the only one solution of $(1.1)_{j}^{\prime}$ with $(1.2)_{j}^{\prime}$.

Namely, we do not encounter the following types of the equilibrium point $(0,0) \in \mathbb{R}^{2}$ :

Non-degenerate (or two-tangent) node: In this case, there are distinct real eigenvalues $\lambda_{+}>\lambda_{-}$of the same sign.

Degenerate node: This is the case where two-tangent node degenerates to $\lambda_{+}=\lambda_{-} \in \mathbb{R} \backslash 0$; it is a stellar node (resp. a one-tangent node) if the matrix in normal form is diagonal (resp. triangular and not diagonal).

Focus: In this case, the eigenvalues $\lambda_{ \pm}$are not real nor purely imaginary.

One-dimensional flow: This is the case where the matrix in normal form is diagonal and of rank one.

Therefore, it suffices to consider the saddle point case $j=0$.

(B). The simplest model. To consider the case $j=0$ amounts to replacing the principal symbol by its simplest model

$$
p^{0}(y, \eta)=\eta_{0}^{2}-y_{0}^{2} \quad \text { in } \quad \mathbb{R}^{n+1} \times \mathbb{R}^{n+1} .
$$


In this case, we have an obvious factorization

$$
p^{0}(y, \eta)=\left(\eta_{0}-y_{0}\right)\left(\eta_{0}+y_{0}\right)=p_{+}^{0}(y, \eta) p_{-}^{0}(y, \eta)
$$

it is then immediately seen that the singular trajectories constitute two straight lines. Indeed, on the trajectory of each bicharacteristic $\rho^{0}=\rho^{0}(s)=$ $(y, \eta)$ of $p^{0}$, we have a Hamilton system with Hamilton function $p_{+}^{0}$ or $p_{-}^{0}$ :

$$
\begin{aligned}
& \frac{d}{d t}\left(y_{0}, \eta_{0}\right)(t)=\left(\frac{\partial p_{ \pm}^{0}}{\partial \eta_{0}},-\frac{\partial p_{ \pm}^{0}}{\partial y_{0}}\right)=(1, \pm 1) \in \mathbb{R}^{2} \\
& \frac{d}{d t}\left(y^{\prime}, \eta^{\prime}\right)(t)=\left(\frac{\partial p_{ \pm}^{0}}{\partial \eta^{\prime}},-\frac{\partial p_{ \pm}^{0}}{\partial y^{\prime}}\right)=(0,0) \in \mathbb{R}^{n} \times \mathbb{R}^{n}
\end{aligned}
$$

Solving it under the initial condition $\rho^{0}(0)=(0,0) \in \boldsymbol{R}^{2 n+2}$, we obtain

$$
\left(y_{0}, \eta_{0}\right)=(t, \pm t), \quad\left(y^{\prime}, \eta^{\prime}\right)=(0,0) \in \mathbb{R}^{n} \times \boldsymbol{R}^{n}
$$

Here, the change of parameters $s \rightarrow t=t^{ \pm}$satisfies the relation

$$
|t|=e^{-2|s|} \text { as } t \rightarrow 0 \text { along singular trajectories. }
$$

We wish to emphasize that, passing from $p^{0}$ to $p_{ \pm}^{0}$, we can take $y_{0}$ as a new parameter $t=t^{ \pm}$.

In the case of quadratic model (i.e. the linearized problem), we have a similar factorization

$$
\begin{aligned}
& p_{\hat{\rho}}(y, \eta)=\left(\eta_{0}-E\left(y_{0}, v\right)^{1 / 2}\right)\left(\eta_{0}+E\left(y_{0}, v\right)^{1 / 2}\right), \\
& \text { where } v=\left(y^{\prime}, \eta^{\prime}\right), E\left(y_{0}, v\right)=y_{0}^{2}+\sum_{j=1}^{n} Q_{j}\left(\tilde{\rho}_{j}, \tilde{\rho}_{j}\right),
\end{aligned}
$$

which does not hold in a full neighborhood of $\tilde{\rho}=(y, \eta)=(0,0)$; it is only valid in a restricted part $y_{0} \neq 0$ (or $|v| /\left|y_{0}\right|<$ constant). Nevertheless, this is sufficient for our purpose, because every incoming or outgoing bicharacteristic $\tilde{\rho}(s)=(y, \eta)$ satisfies $v=0$-we have

$$
\frac{d}{d s}\left(y_{j}^{2}+\eta_{j}^{2}\right)=0 \quad \text { for } \quad 1 \leq j \leq n
$$


Furthermore, the term $E\left(y_{0}, v\right)^{1 / 2}$ in this model does not involve the variable $\eta_{0}$, so that as in the simplest case we can single out $y_{0}$ as a new parameter:

$$
\left.\frac{d}{d t^{ \pm}} y_{0}\left(t^{ \pm}\right)=1 \quad \text { (thus, we may take } t=t^{ \pm}=y_{0}\right)
$$

Therefore, we obtain a closed system having $v=v(t)$ as unknown, a system which admits only a trivial solution $v(\cdot)=0$ (under the initial condition $v(0)=0$ ), as we have already known.

In what follows, we shall observe that the general problem can be put in a situation similar to that as above.

\subsection{How to continue bicharacteristics}

In order to prove Theorem 1 above, we shall perform in Subsection 2.1 below a symplectic change of variables about $\rho=\hat{\rho}$. In the new coordinates $\rho=(y, \eta)$ with $\hat{\rho}=(0,0)$, the principal symbol $p=p(\rho)$ takes the form

$$
p(\rho)=q(\rho)\left[\left\{\eta_{0}-\varphi\left(y, \eta^{\prime}\right)\right\}^{2}-\psi\left(y, \eta^{\prime}\right)\right] \text { with } \quad q(\hat{\rho}) \neq 0,
$$

so that we may assume $q(\rho)=1$. Here, $p(y, \cdot)$ is hyperbolic with respect to $d y_{0}$ near $y=0$, a fact which implies that

$$
\psi\left(\rho^{*}\right) \geq 0 \text { near } \rho^{*}=\left(y, \eta^{\prime}\right)=(0,0) \in \mathbb{R}^{2 n+1} \text {. }
$$

Furthermore, if we write $O^{l}=O\left(\left|\rho^{*}\right|^{l}\right)$ as $\rho^{*} \rightarrow 0$, then

$$
\varphi\left(\rho^{*}\right)=O^{2}, \psi\left(\rho^{*}\right)-E\left(\rho^{*}\right)=O^{3}, \quad \text { where }
$$

$$
E\left(\rho^{*}\right)=y_{0}^{2}+\sum_{j=1}^{n_{1}} \mu_{j}\left(y_{j}^{2}+\eta_{j}^{2}\right)+\mu_{0} \sum_{j=n_{1}+1}^{n_{2}} y_{j}^{2}
$$

with some positive constants $\mu_{j}, \mu_{0}$, where $0 \leq n_{1} \leq n_{2} \leq n$. (The case $n_{1}=n_{2}=n$ does not occur in our problem, though we need not exclude it in what follows.)

We now set, for $u=\left(z^{\prime}, \zeta^{\prime}\right) \in \mathbb{R}^{2 n}$ with $|u|<1$, that

$$
\Phi\left(y_{0}, u\right)=y_{0}^{-2} \varphi\left(y_{0}, y_{0} u\right), \quad \Psi\left(y_{0}, u\right)=y_{0}^{-2} \psi\left(y_{0}, y_{0} u\right),
$$




$$
\pi^{ \pm}\left(y_{0}, u\right)= \pm \Psi\left(y_{0}, u\right)^{1 / 2}+y_{0} \Phi\left(y_{0}, u\right)
$$

where the square root can be taken by virtue of (1.4). Observe that $\Phi$ and $\Psi$ are smooth (i.e. $C^{\infty}$ or analytic, corresponding to the assumption on the principal symbol $p=p(\rho))$ as far as $\left|y_{0}\right|$ is small, a fact which can be observed by inspecting the remainders of the Taylor expansions. Similarly for $\Psi^{1 / 2}$, a consequence of the fact $\Psi\left(y_{0}, u\right)=E(1, u)+\cdots$. Thus, the functions $\pi^{ \pm}$ are smooth whenever $\left|y_{0}\right|$ is small, say, in $\left\{\left|y_{0}\right|<a,|u|<1\right\}$, and satisfy

$$
\pm \pi^{ \pm}(0, u)=E(1, u)^{1 / 2}=1+\frac{1}{2} E(0, u)+O\left(|u|^{4}\right)
$$

as $u \rightarrow 0$. Therefore, setting $v=\left(y^{\prime}, \eta^{\prime}\right)$ and

$$
p^{ \pm}(y, \eta)=\eta_{0}-y_{0} \pi^{ \pm}\left(y_{0}, v / y_{0}\right)
$$

we have a factorization of the principal symbol

$$
p(\rho)=p^{+}(\rho) p^{-}(\rho) \quad \text { near } \quad \rho=\hat{\rho}
$$

which is valid within a certain cone (or, rather, a two-sided wedge having the $\eta_{0}$-axis as its edge):

$$
|v|=\left|\left(y^{\prime}, \eta^{\prime}\right)\right|<a\left|y_{0}\right| \quad \text { with some constant } a>0
$$

It will be shown in Subsection 2.2 below that:

Proposition 1. Suppose given an incoming or outgoing bicharacteristic $\rho(s)=(y(s), \eta(s))$, a solution of (1.1) with (1.2). Then, $v(s)=\left(y^{\prime}(s), \eta^{\prime}(s)\right)$ satisfies

$$
|v(s)| \leq C\left|y_{0}(s)\right|^{3 / 2} \quad \text { as } \quad \rho(s) \rightarrow \hat{\rho},
$$

where $C>0$ is a constant independent of the choice of a bicharacteristic $\rho=\rho(s)$.

By virtue of Proposition 1 above, every solution of (1.1) with (1.2) must stay, locally near $\rho=\hat{\rho}$, in the cone (1.9). Furthermore, we see, in view of the factorization (1.8), that it must be an integral curve $\rho=\rho(t)$ of either $H_{p^{+}}$or $H_{p^{-}}$with the limit point $\rho=\hat{\rho}$, where the parameter must be changed from $s$ to $t=t^{ \pm}$. Therefore, setting

$$
\rho(t)=(y(t), \eta(t)), \quad v(t)=\left(y^{\prime}(t), \eta^{\prime}(t)\right),
$$


we have $\left(d y_{0} / d t\right)(t)=1$ in either case, so that we may take $y_{0}$ to be the parameter $t=t^{ \pm}$simultaneously. Then,

$$
\frac{d y^{\prime}}{d t}(t)=-\frac{\partial \pi^{ \pm}}{\partial \zeta^{\prime}}\left(t, \frac{v(t)}{t}\right), \quad \frac{d \eta^{\prime}}{d t}=\frac{\partial \pi^{ \pm}}{\partial z^{\prime}}\left(t, \frac{v(t)}{t}\right)
$$

$$
\frac{v(t)}{t} \rightarrow 0 \in \mathbb{R}^{2 n} \quad \text { as } \quad t \rightarrow 0 \quad \text { (by Proposition 1), }
$$

and similarly for the scalar function $\eta_{0}=\eta_{0}(t)$, that is,

$$
(1.12)^{ \pm} \quad \frac{d \eta_{0}}{d t}(t)=\pi^{ \pm}\left(t, \frac{v(t)}{t}\right)-\frac{v(t)}{t} \cdot \frac{\partial \pi^{ \pm}}{\partial u}\left(t, \frac{v(t)}{t}\right), \quad \eta_{0}(0)=0
$$

Conversely, if we are given a solution of $(1.11)^{ \pm}$, then we can reproduce a solution of (1.1) with (1.2) by integrating $(1.12)^{ \pm}$.

It may be worthwhile noting that (1.7) with (1.6) determines how the orientation of the parameter is changed from (1.1) to (1.11) ${ }^{ \pm}$. Namely, in case $q>0$ in (1.3), every solution of $(1.11)^{+}$(resp. $\left.(1.11)^{-}\right)$in either $t>0$ or $t<0$ corresponds to an incoming (resp. outgoing) bicharacteristic of $p$. The situation is reversed in case $q<0$.

We are thus led to investigate the unique existence and the regularity for the solution of each one of the initial value problems $(1.11)^{ \pm}$. Here, the uniqueness is for the one-sided problems, whereas the regularity is for the two-sided ones. Evidently, the regularity of $v=v(t)$ in $(1.11)^{ \pm}$ensures that of $\eta_{0}=\eta_{0}(t)$ in $(1.12)^{ \pm}$.

Let us write $(1.11)^{ \pm}$as follows:

$$
\frac{d v}{d t}(t)=F\left(t, \frac{v(t)}{t}\right), \quad \frac{v(t)}{t} \rightarrow 0 \in \mathbb{R}^{N} \quad \text { as } \quad t \rightarrow 0
$$

where $N=2 n$ and

$$
F\left(y_{0}, u\right)=F^{ \pm}\left(y_{0}, u\right)=\left(-\frac{\partial \pi^{ \pm}}{\partial \zeta^{\prime}}\left(y_{0}, u\right), \frac{\partial \pi^{ \pm}}{\partial z^{\prime}}\left(y_{0}, u\right)\right)
$$

It then follows from (1.6) that 


$$
F(0,0)=0, \quad \sigma\left(F_{u}(0,0)\right) \subset\{\lambda \in \mathbb{C} ; \quad \operatorname{Re} \lambda \leq 0\},
$$

where $\sigma\left(F_{u}(0,0)\right)$ stands for the spectrum of the Jacobian matrix $F_{u}(0,0)$ $=(\partial F / \partial u)(0,0)$. Indeed,

$$
\operatorname{det}\left(\mathscr{H}-\lambda I_{2 n+2}\right)=\left(\lambda^{2}-1\right) \operatorname{det}\left(F_{u}^{ \pm}(0,0)-\lambda I_{2 n}\right),
$$

so that the eigenvalues of each one of $F_{u}^{ \pm}(0,0)$ are 0 and $\pm \sqrt{-1} \mu_{j}$ for $j=1,2, \cdots, n_{1}$. We shall prove in Section 3 below that:

Theorem 2. Suppose given a $C^{\infty}$ (resp. analytic) function $F=F\left(y_{0}, u\right)$ $\in \mathbb{R}^{N}$ near $\left(y_{0}, u\right)=(0,0) \in \mathbb{R} \times \mathbb{R}^{N}$ satisfying (1.13). Then, the two-sided initial value problem (1.11) admits a $C^{\infty}$ (resp. analytic) solution $v=v(t)$ near $t=0$. Furthermore, the uniqueness is valid for each one of the one-sided problems, among solutions of $C^{1}$-class except at the end point $t=0$.

Therefore, Theorem 1 will be established if we shall prove Theorem 2 together with Proposition 1.

Remark. In view of $(1.11)^{ \pm}$and $(1.12)^{ \pm}$, we see that

$$
\frac{d v}{d t}(0)=0, \quad \frac{d \eta_{0}}{d t}(0)=\pi^{ \pm}(0,0)= \pm 1
$$

so that the integral curves $\rho=\rho^{ \pm}(t)$ of $H_{p^{ \pm}}$with $\rho^{ \pm}(0)=\hat{\rho}$ satisfy, respectively,

$$
(1.14)^{ \pm} \quad \mathscr{H} \frac{d \rho^{ \pm}}{d t}(0)= \pm \frac{d \rho^{ \pm}}{d t}(0)
$$

Namely, the tangent lines at $\hat{\rho}$ of the two curves in Theorem 1 are spanned by eigenvectors of the Hamilton map $\mathscr{H}$ associated with the two non-vanishing real eigenvalues $\pm \lambda$. In $(1.14)^{ \pm}$above, the normalization $\lambda=1$ is made corresponding to the assumption $q(\rho)=1$ in (1.3).

\section{$\S 2$. Preliminary Reductions}

\subsection{Normalization by symplectic transformations}

The present subsection is devoted to the justification of (1.3) with (1.4) 
and (1.5) stated in Subsection 1.3. Every function will be $C^{\infty}$ or analytic near $\rho=\hat{\rho}$, without any mention of it, corresponding to the assumption on the principal symbol $p=p(\rho)$. In particular, the abbreviated expression $O^{l}$ as in (1.5) can be differentiated in a usual manner.

We first reduce the problem to the case of second order symbol $p=p(\rho)$. More precisely,

Lemma 2.1. After a symplectic change of coordinates about $\rho=\hat{\rho}$, the principal symbol takes the form

$$
p(x, \xi)=q(x, \xi)\left\{\xi_{0}^{2}-\psi\left(x, \xi^{\prime}\right)\right\}
$$

where $q(\hat{x}, \hat{\xi}) \neq 0$ and $\psi\left(x, \xi^{\prime}\right) \geq 0$.

Proof. We first observe that

$$
\frac{\partial^{2} p}{\partial \xi_{0}^{2}}(\hat{\rho}) \neq 0
$$

a fact which is obtained without difficulty by using Lemma 1.3.1 in Hörmander [5]. Namely, suppose the contrary; then the hyperbolicity implies the vanishing of the Hessian of $p(\rho)$ at $\rho=\hat{\rho}$, but this contradicts the effective hyperbolicity.

By virtue of (2.2), we may apply the Malgrange (or Weierstrass) preparation theorem and conclude that $p(x, \xi)$ takes the form

$$
\begin{aligned}
& p(x, \xi)=q(x, \xi) \tilde{p}(x, \xi), \quad q(\hat{x}, \xi) \neq 0 \\
& \tilde{p}(x, \xi)=\left\{\xi_{0}-\tilde{\varphi}\left(x, \xi^{\prime}\right)\right\}^{2}-\tilde{\psi}\left(x, \xi^{\prime}\right) .
\end{aligned}
$$

Here, the hyperbolicity inherits from $p(x, \xi)$ to $\tilde{p}(x, \xi)$, so that $\tilde{\psi}\left(x, \xi^{\prime}\right) \geq 0$.

The desired symplectic coordinate system is obtained by a repeated application of the Frobenius theorem (see Theorem 21.1.6 in Hörmander [6]). Indeed, it is possible to construct a change of variables $(x, \xi) \rightarrow(\tilde{x}, \tilde{\xi})$ :

$$
\begin{array}{ll}
\tilde{\xi}_{0}=\xi_{0}-\tilde{\varphi}\left(x, \xi^{\prime}\right), & \tilde{\xi}^{\prime}=\tilde{\xi}^{\prime}\left(x, \xi^{\prime}\right), \\
\tilde{x}_{0}=x_{0}, & \tilde{x}^{\prime}=\tilde{x}^{\prime}\left(x, \xi^{\prime}\right),
\end{array}
$$

in such a way that, for $0 \leq j, k \leq n$, 


$$
\left\{\tilde{\xi}_{j}, \tilde{\xi}_{k}\right\}=\left\{\tilde{x}_{j}, \tilde{x}_{k}\right\}=0, \quad\left\{\tilde{\xi}_{j}, \tilde{x}_{k}\right\}=\delta_{j k},
$$

where $\{\because, \cdot\}$ stands for the Poisson bracket.

q.e.d.

Remark. The symplectic transformation in Lemma 2.1 above can be taken to preserve the homogeneity (cf. Theorem 21.1.9 in Hörmander [6]). Furthermore, it is possible to make $\tilde{p}(x, \xi)$ homogeneous in $\xi$ in the proof above. Then, $\psi\left(x, \xi^{\prime}\right)$ in $(2.1)$ is homogeneous of degree two in $\xi^{\prime}$, so that the problem is completely reduced to the case $m=2$. In what follows, however, we shall not use the homogeneity of $\psi\left(x, \xi^{\prime}\right)$.

Without loss of generality, we assume that $q(x, \xi)=1$ in (2.1). It is also convenient to deal with a coordinate system having $\hat{\rho}$ as the origin because the homogeneity is not concerned. We thus introduce a coordinate system $\rho=(y, \eta)$ by setting

$$
(y, \eta)=(x-\hat{x}, \xi-\xi) \in \mathbb{R}^{2 n+2},
$$

so that $\hat{\rho}=(0,0)$. Then,

Lemma 2.2. After a linear symplectic change of coordinates about $\rho=\hat{\rho}$, the symbol $p=p(y, \eta)$ normalized as above takes the form

$$
p(y, \eta)=c\left\{\eta_{0}^{2}-E\left(y, \eta^{\prime}\right)\right\}+O^{3}(y, \eta) \quad \text { as } \quad \rho \rightarrow \hat{\rho},
$$

with some constant $c>0$, where $E\left(y, \eta^{\prime}\right)$ is a quadratic polynomial of $\left(y, \eta^{\prime}\right)$ given by (1.5) with $\rho^{*}=\left(y, \eta^{\prime}\right)$. Furthermore, preserved is the hyperbolicity of $p(y, \cdot)$ with respect to $d y_{0}$ near $y=0$, after the change of coordinates.

Proof. Let us first write (2.1) in Lemma 2.1 with $q(x, \xi)=1$ in terms of the $(y, \eta)$ coordinates as follows:

$$
p(y, \eta)=\left(\eta_{0}+\xi_{0}\right)^{2}-\psi\left(y, \eta^{\prime}\right), \quad \psi\left(y, \eta^{\prime}\right) \geq 0 .
$$

Since $\hat{\rho} \in \Sigma_{2}$, it follows that $\hat{\xi}_{0}=0$. We next set

$$
p_{\hat{\rho}}(y, \eta)=Q((y, \eta),(y, \eta))=\eta_{0}^{2}-\psi_{\hat{\rho}}\left(y, \eta^{\prime}\right),
$$

where $Q(\cdot, \cdot)$ is a quadratic polynomial as in Subsection 1.1, so that

$$
p(y, \eta)=p_{\hat{\rho}}(y, \eta)+O^{3}(y, \eta) \quad \text { as } \quad \rho \rightarrow \hat{\rho} .
$$


By virtue of the effective hyperbolicity, we can write $p_{\hat{\rho}}(y, \eta)$ in normal form (see, e.g., Theorem 21.5.3 of Hörmander [6]) as follows: there exists a linear symplectic change of coordinates $S:(\tilde{y}, \tilde{\eta}) \mapsto(y, \eta)$ about $(0,0)$ such that

$$
p_{\hat{\rho}}(y, \eta)=p_{\hat{\rho}}(S(\tilde{y}, \tilde{\eta}))=c\left\{\tilde{\eta}_{0}^{2}-E\left(\tilde{y}, \tilde{\eta}^{\prime}\right)\right\}
$$

with some constant $c>0$, where $E\left(\tilde{y}, \tilde{\eta}^{\prime}\right)$ is given by (1.5) with $\rho^{*}=$ $\left(\tilde{y}, \tilde{\eta}^{\prime}\right)$. Setting

$$
\tilde{p}(\tilde{y}, \tilde{\eta})=p(S(\tilde{y}, \tilde{\eta}))=p(y, \eta)
$$

we see that its quadratic part is given by

$$
\tilde{p}_{\hat{\rho}}(\tilde{y}, \tilde{\eta})=p_{\hat{\rho}}(S(\tilde{y}, \tilde{\eta}))=p_{\hat{\rho}}(y, \eta) .
$$

Therefore, we obtain $(2.3)$ in the $(\tilde{y}, \tilde{\eta})$ coordinates.

It remains to observe that the hyperbolicity with respect to the $\eta_{0}$ variable is preserved under the symplectic transformation $S$ as above. Since $S$ is linear, this fact is easily verified as follows. Setting

$$
\tilde{p}_{ \pm}(\tilde{y}, \tilde{\eta})=p_{ \pm}(y, \eta)=\eta_{0} \mp \psi\left(y, \eta^{\prime}\right)^{1 / 2}
$$

we shall show that each one of $\tilde{p}_{ \pm}(\tilde{y}, \tilde{\eta})$ has a real zero

$$
\tilde{\eta}_{0}^{ \pm}=\tilde{\eta}_{0}^{ \pm}\left(\tilde{y}, \tilde{\eta}^{\prime}\right) \quad \text { with } \quad\left|\tilde{\eta}_{0}^{ \pm}\right| \leq \varepsilon
$$

in a closed region $\left|\left(\tilde{y}, \tilde{\eta}^{\prime}\right)\right| \leq \varepsilon^{2}$, as far as the constant $\varepsilon>0$ is small enough. (It should be noticed that $p_{ \pm}$above are different from $p^{ \pm}$in Subsection 1.3). If we write

$$
\eta_{0}=\alpha \tilde{\eta}_{0}+O\left(\varepsilon^{2}\right), \psi\left(y, \eta^{\prime}\right)=\beta \tilde{\eta}_{0}^{2}+O\left(\varepsilon^{3}\right)
$$

in a closed region $\left\{(\tilde{y}, \tilde{\eta}) ;\left|\tilde{\eta}_{0}\right| \leq \varepsilon,\left|\left(\tilde{y}, \tilde{\eta}^{\prime}\right)\right| \leq \varepsilon^{2}\right\}$ with constants $\alpha$ and $\beta$, then $\beta \geq 0$ and

$$
\tilde{p}(\tilde{y}, \tilde{\eta})=p(y, \eta)=\left(\alpha^{2}-\beta\right) \tilde{\eta}_{0}^{2}+O\left(\varepsilon^{3}\right),
$$

so that (2.3) yields $\alpha^{2}-\beta=c>0$. On the other hand, if in addition $\left|\tilde{\eta}_{0}\right|=\varepsilon$ then

$$
\tilde{p}_{ \pm}(\tilde{y}, \tilde{\eta})=\alpha \tilde{\eta}_{0} \mp \sqrt{\beta}\left|\tilde{\eta}_{0}\right|+O\left(\varepsilon^{2}\right)
$$

so that each one of $\tilde{p}_{ \pm}(\tilde{y}, \tilde{\eta})$ has different sign at $\tilde{\eta}_{0}= \pm \varepsilon$. Therefore, the 
desired conclusion follows from the continuity of $\tilde{p}_{ \pm}(\tilde{y}, \tilde{\eta})$ with respect to the $\tilde{\eta}_{0}$ variable.

q.e.d.

Justification of (1.3) with (1.4) and (1.5). By virtue of Lemmas 2.1 and 2.2 , we have, after a symplectic change of coordinates about $\rho=\hat{\rho}$, that

$$
\begin{aligned}
& p(y, \eta)=\tilde{q}(y, \eta)\left\{\eta_{0}^{2}-\tilde{\psi}(y, \eta)\right\}, \\
& \tilde{\psi}(y, \eta)=E\left(y, \eta^{\prime}\right)+O^{3}(y, \eta) \quad \text { as } \quad \rho \rightarrow \hat{\rho},
\end{aligned}
$$

where $\tilde{q}(\hat{\rho}) \neq 0$. It then follows from the Malgrange (or Weierstrass) preparation theorem that $p(y, \eta)$ takes the form (1.3) in which $\varphi(0,0)$ $=\psi(0,0)=0$. Comparing the quadratic parts of these two expressions of the same function $p=p(y, \eta)$, we obtain (1.5). Let us finally recall by Lemma 2.2 that $p(y, \cdot)$ in the new coordinates is hyperbolic with respect to $d y_{0}$ near $y=0$. This together with (1.3) implies (1.4).

2.2. An estimate for bicharacteristics near the reference point

The purpose of the present subsection is to prove Proposition 1. Let us begin with the following simple observation.

Lemma 2.3. There exists a constant $C>0$ such that

$$
|d p(\rho)| \leq C\left|\frac{\partial p}{\partial \eta_{0}}(\rho)\right| \text { for } \quad \rho \in \Sigma \quad \text { near } \hat{\rho}
$$

Proof. If $\rho \in \Sigma$, then $p(\rho)=0$, so that

$$
\psi\left(\rho^{*}\right)^{1 / 2}=\left|\eta_{0}-\varphi\left(\rho^{*}\right)\right|=\frac{1}{2}\left|\frac{\partial p}{\partial \eta_{0}}(\rho)\right| .
$$

On the other hand, $\psi \geq 0$ implies $|d \psi| \leq C^{\prime} \psi^{1 / 2}$ with some constant $C^{\prime}>0$. Therefore, the desired conclusion follows from

$$
\frac{\partial p}{\partial y}=2\left(\varphi-\eta_{0}\right) \frac{\partial \varphi}{\partial y}-\frac{\partial \psi}{\partial y}, \quad \frac{\partial p}{\partial \eta^{\prime}}=2\left(\varphi-\eta_{0}\right) \frac{\partial \varphi}{\partial \eta^{\prime}}-\frac{\partial \psi}{\partial \eta^{\prime}}
$$

q.e.d.

By using Lemma 2.3, we obtain the following estimate for incoming 
or outgoing bicharacteristic $\rho=\rho(s)$.

Lemma 2.4. A weaker version of Proposition 1 holds, where the conclusion (1.10) is replaced by

$$
|\rho(s)| \leq C\left|y_{0}(s)\right| \quad \text { as } \quad \rho(s) \rightarrow \hat{\rho} .
$$

Proof. We first note that

$$
\dot{y}_{0}(s) \neq 0 \text { as } \rho(s) \rightarrow \hat{\rho},
$$

where the dot refers to differentiation with respect to $s$. Indeed, if $\dot{y}_{0}(s)=0$ at some point $\rho(s) \in \Sigma_{1}$ near $\hat{\rho}$, then

$$
\frac{\partial p}{\partial \eta_{0}}(\rho(s))=\dot{y}_{0}(s)=0
$$

so that Lemma 2.3 implies $d p(\rho(s))=0$, but this contradicts $\rho(s) \in \Sigma_{1}$. Thus, we may take $y_{0}$ as a new parameter of the curve $\rho=\rho(s)$ near the reference point $\hat{\rho}$. Then,

$$
\dot{\rho}(s)=\dot{y}_{0}(s) \frac{d}{d y_{0}} \rho\left(s\left(y_{0}\right)\right)
$$

so that Lemma 2.3 implies

$$
\left|\frac{\partial p}{\partial \eta_{0}} \frac{d}{d y_{0}} \rho\right|=\left|<H_{p}, d \rho>\right|=|d p| \leq C\left|\frac{\partial p}{\partial \eta_{0}}\right|
$$

on the curve $\rho=\rho(s)$. Therefore,

$$
|\rho(s)|=\left|\int_{0}^{y_{0}(s)} \frac{d}{d y_{0}} \rho\left(s\left(y_{0}\right)\right) d y_{0}\right| \leq C\left|y_{0}(s)\right| .
$$

By virtue of Lemma 2.4 above, it can be understood that

$$
O^{l}=O^{l}(y, \eta)=O\left(\left|y_{0}\right|^{l}\right) \quad \text { as } \quad \rho \rightarrow \hat{\rho}
$$

as far as the limit is taken along a bicharacteristic.

The following lemma is crucial in the proof of Proposition 1. 
Lemma 2.5. For every $\varepsilon>0$, there exists $\delta>0$ such that

$$
\left|y_{0}(s)\right| \leq \frac{1+\varepsilon}{2}\left|\dot{y}_{0}(s)\right| \text { whenever } 0<\left|y_{0}(s)\right|<\delta
$$

where $\delta$ is independent of the choice of a bicharacteristic $\rho=\rho(s)$ satisfying $\rho(s) \rightarrow \hat{\rho}$.

Proof. We shall be working on the bicharacteristic $\rho=\rho(s)$. Since the trajectory is included in the characteristic set $\Sigma$, it follows that

$$
\left|\dot{y}_{0}\right|=\left|\frac{\partial p}{\partial \eta_{0}}(\rho)\right|=2\left|\eta_{0}-\varphi\left(\rho^{*}\right)\right|=2 \psi\left(\rho^{*}\right)^{1 / 2} .
$$

On the other hand, we have by using (1.5) and Lemma 2.4 that

$$
y_{0}^{2} \leq E\left(\rho^{*}\right)=\psi\left(\rho^{*}\right)+O^{3}
$$

under the notation (2.4). Since $\left|y_{0}\right|$ is small enough, we obtain the desired conclusion (2.5).

q.e.d.

Remarks. (1) In proving Proposition 1, we only need the fact that $(1+\varepsilon) / 2$ in $(2.5)$ above is a positive constant independent of the choice of a bicharacteristic. Nevertheless, it might be worthwhile noting that (2.5) yields the following estimate

$$
Y_{+}(s) \leq Y_{+}\left(s_{0}\right) \quad \text { as } \quad \rho(s) \rightarrow \hat{\rho} \quad \text { with } s_{0} \text { fixed, }
$$

where $Y_{ \pm}(s)=\left|y_{0}(s)\right| \exp [2|s| /(1 \pm \varepsilon)]$. In particular,

$$
\left|y_{0}(s)\right| \leq C \exp \left(\frac{-2}{1+\varepsilon}|s|\right) \text { as } \rho(s) \rightarrow \hat{\rho}
$$

with some constant $C>0$, cf. Subsection 1.2 , (B).

(2) A reversed inequality with $1-\varepsilon$ in place of $1+\varepsilon$ is also obtained by using the conclusion of Proposition 1 (or Theorem 2). Namely, it follows that $v=O^{3 / 2}$ (or $v=O^{2}$ ) under the notation (2.4), so that $\psi\left(\rho^{*}\right)-y_{0}^{2}=O^{3}$. This yields, as in the proof of Lemma 2.5 , that

$$
\left|y_{0}(s)\right| \geq \frac{1-\varepsilon}{2}\left|\dot{y}_{0}(s)\right| \quad \text { whenever } \quad 0<\left|y_{0}(s)\right|<\delta
$$


which in turn leads to

$$
Y_{-}(s) \geq Y_{-}\left(s_{0}\right) \quad \text { as } \quad \rho(s) \rightarrow \hat{\rho} \quad \text { with } s_{0} \text { fixed. }
$$

In particular, with some constant $C>0$,

$$
\left|y_{0}(s)\right| \geq C \exp \left(\frac{-2}{1-\varepsilon}|s|\right) \text { as } \rho(s) \rightarrow \hat{\rho} .
$$

\section{Proof of Proposition 1. Settintg}

$$
e_{j}(s)=\frac{1}{2}\left\{y_{j}(s)^{2}+\eta_{j}(s)^{2}\right\} \quad \text { for } \quad 1 \leq j \leq n
$$

we shall show under the notation (2.4) that

$$
e_{j}=O^{3} \text { for } 1 \leq j \leq n_{1}, \quad e_{j}=O^{4} \text { for } n_{1}<j \leq n \text {. }
$$

Let us first consider the case $1 \leq j \leq n_{1}$. Since

$$
\frac{\partial p}{\partial y_{j}}(\rho)=2 \mu_{j} y_{j}+O^{2}, \quad \frac{\partial p}{\partial \eta_{j}}(\rho)=2 \mu_{j} \eta_{j}+O^{2}
$$

under the notation (2.4), it follows that

$$
\dot{e}_{j}(s)=y_{j} \frac{\partial p}{\partial \eta_{j}}(\rho)-\eta_{j} \frac{\partial p}{\partial y_{j}}(\rho)=O^{3}
$$

This together with Lemma 2.5 implies

$$
\frac{d}{d y_{0}} e_{j}\left(s\left(y_{0}\right)\right)=O^{2}
$$

where a change of variables $s \rightarrow y_{0}$ is made as in the proof of Lemma 2.4. Integrating both sides with respect to $y_{0}$, we obtain the desired estimate $e_{j}=O^{3}$.

In case $n_{2}<j \leq n$, we have

$$
\dot{y}_{j}(s)=\frac{\partial p}{\partial \eta_{j}}(\rho)=O^{2}, \quad \dot{\eta}_{j}(s)=-\frac{\partial p}{\partial y_{j}}(\rho)=O^{2}
$$


so that $e_{j}=O^{4}$ is obtained by the same argument as above. Indeed,

$$
\frac{d}{d y_{0}} y_{j}\left(s\left(y_{0}\right)\right)=O^{1}, \quad \frac{d}{d y_{0}} \eta_{j}\left(s\left(y_{0}\right)\right)=O^{1} .
$$

It remains to consider the case $n_{1}<j \leq n_{2}$. We first have, as above,

$$
\dot{y}_{j}(s)=\frac{\partial p}{\partial \eta_{j}}(\rho)=O^{2}, \quad \frac{d}{d y_{0}} y_{j}\left(s\left(y_{0}\right)\right)=O^{1},
$$

so that $y_{j}=O^{2}$. By using this, we obtain

$$
\dot{\eta}_{j}(s)=-\frac{\partial p}{\partial y_{j}}(\rho)=-2 \mu_{0} y_{j}+O^{2}=O^{2},
$$

so that $\eta_{j}=O^{2}$, and hence $e_{j}=O^{4}$. This completes the proof of (2.6).

\section{$\S$ 3. A Singular Initial Value Problem}

\subsection{The Briot-Bouquet singularity}

In order to prove Theorem 2, we begin with the existence result in the analytic case. Setting $u(t)=v(t) / t$, we write (1.11) as

$$
t \frac{d u}{d t}(t)=f(t, u(t)), \quad u(0)=0 \in \boldsymbol{R}^{N}
$$

with $f(t, u)=F(t, u)-u$, so that (1.13) implies

$$
f(0,0)=0, \sigma(M) \subset\{\lambda \in C ; \operatorname{Re} \lambda<0\},
$$

where $M=f_{u}(0,0)$. Let us now recall a celebrated result of Briot and Bouquet in [1] which states that:

Proposition 2. If $f=f(t, u) \in C^{N}$ is holomorphic near $(t, u)=(0,0) \in C \times C^{N}$ and satisfies

$$
f(0,0)=0, \quad \sigma(M) \cap N=\varnothing,
$$


where $\mathbb{N}=\{1,2, \cdots\}$, then (3.1) admits a unique holomorphic solution $u=u(t)$ near $t=0 \in \mathbb{C}$.

Indeed, Proposition 2 in case $N=1$ is obtained in [1], pp. 164-171 (see also, e.g., Ince [8] or Hille [2]), and their method remains valid for general $N$. The proof is sketched as follows.

It is easily seen that the assumption (3.2)' implies the unique existence of a solution as a formal power series $u(t)=c_{1} t+c_{2} t^{2}+\cdots$. Thus, it suffices to find a convergent scalar majorant $U(t)=C_{1} t+C_{2} t^{2}+\cdots$, which can be obtained as the unique solution of a functional equation of the form

$$
\varepsilon U(t)=\Phi(t, U(t)), \quad U(0)=0 \in \mathbb{C}
$$

with a small constant $\varepsilon>0$, where $\Phi(t, U)$ is a standard scalar majorant of $f(t, u)-M u$. Since $\Phi(0,0)=\Phi_{U}(0,0)=0$, the unique existence of a holomorphic solution $U(t)$ of $(3.1)^{+}$near $t=0 \in \mathbb{C}$ follows from the implicit function theorem.

Remark. It turns out that every formal solution of (3.1) is necessarily convergent without any assumption on $\sigma(M)$, cf. Hukuhara et al. [7], pp. 77-78. Indeed, the subtraction of the polynomial of degree $\leq r$ part $c_{1} t+\cdots+c_{r} t^{r}$ from $u(t)$ amounts to reducing the eigenvalues of $M$ by $r$. Taking $r$ large enough, we may apply the contraction principle to the reduced equation in order to obtain a holomorphic solution, while the formal solution of the original equation is unique up to terms of degree $\leq r$. Hence, an arbitrarily prescribed formal solution must be holomorphic near $t=0$.

Therefore, it remains to establish the $C^{\infty}$ version of the Briot-Bouquet theorem (Proposition 2) under the assumption (3.2) in place of (3.2)'. This has been done by de Hoog and Weiss [3], [4] (see also Russell [14]). We shall reproduce the proof in Subsections 3.2 and 3.3 below, because the setting in [3], [4] is too general for our purpose.

The proof of Theorem 2 in the $C^{\infty}$ case is fairly elementary. We shall first consider in Subsection 3.2 a linear problem with "constant coefficients." Then, the nonlinear problem can be regarded as a perturbation of the linear one - as we shall see in Subsection 3.3. Indeed, the unique existence is obtained via the contraction principle, whereas the proof of the regularity requires a closer look at the linear problem. 


\subsection{A linear system}

Let us consider a linear system of singular ordinary differential equations

$$
t \frac{d u}{d t}(t)-M u(t)=g(t) \text { for } 0<t \leq T
$$

where $g \in C^{0}[0, T]=C^{0}\left([0, T], C^{N}\right)$ and $M \in M_{N}(C)$. As in (3.2), we assume that

$$
\sigma(M) \subset\{\lambda \in C ; \quad \operatorname{Re} \lambda<0\}
$$

In order to solve (3.3) in the space $\mathscr{C}_{T}^{0.1}=C^{0}[0, T] \cap C^{1}(0, T]$, let us introduce a linear operator $\mathscr{E}_{M}: C^{0}[0, T] \rightarrow \mathscr{C}_{T}^{0.1}$ by setting

$$
\mathscr{E}_{M} g(t)=\int_{0}^{t}\left(\frac{t}{s}\right)^{M} g(s) \frac{d s}{s}=\int_{0}^{1}\left(\frac{1}{s}\right)^{M} g(t s) \frac{d s}{s}
$$

where $t^{M}=\exp (M \log t)$, so that $\mathscr{E}_{M} g(0)=-M^{-1} g(0)$. It is easily seen that $u=\mathscr{E}_{M} g$ satisfies the equation (3.3). Furthermore,

Lemma 3.1. A unique solution $u \in \mathscr{C}_{T}^{0,1}$ of (3.3) is given by $u=\mathscr{E}_{M} g$.

Proof. With $\tau \in(0, T]$ arbitrarily fixed, the general solution of (3.3) takes the form

$$
u(t)=\left(\begin{array}{l}
t \\
\tau
\end{array}\right)^{M} u(\tau)+\int_{\tau}^{t}\left(\begin{array}{l}
t \\
\frac{-}{s}
\end{array}\right)^{M} g(s) \frac{d s}{s}
$$

If $u \in \mathscr{C}_{T}^{0,1}$, then the assumption (3.4) permits us to take the limit $\tau \downarrow 0$ in (3.5); thus $u=\mathscr{E}_{M} g$.

The differentiability property of the operator $\mathscr{E}_{M}$ is clear from the second expression of its definition. Namely, if $g \in C^{r}[0, T]$ with some $r \in N_{0}=\{0,1$, $2, \cdots\}$, then $\mathscr{E}_{M} g \in \mathscr{C}_{T}^{r, 1}=C^{r}[0, T] \cap C^{r+1}(0, T]$ and

$$
\left(\mathscr{E}_{M} g\right)^{(r)}=\left(\frac{d}{d t}\right)^{r} \mathscr{E}_{M} g=\mathscr{E}_{M-r I} g^{(r)}
$$

where $I \in M_{N}(C)$ stands for the identity matrix. In particular, 


$$
\left(\mathscr{E}_{M} g\right)^{(r)}(0)=(r I-M)^{-1} g^{(r)}(0)
$$

In the application to the nonlinear problem, we also need an estimate for the operators $\mathscr{E}_{M-r I}$ with $r \in N_{0}$ in terms of the norm $\|u\|_{T}=\max \{|u(t)|$; $0 \leq t \leq T\}$ for $u \in C^{0}[0, T]$, where the pointwise norm $|\cdot|$ on $\mathbb{C}^{N}$ is specified appropriately. The following lemma asserts that the operator norm of $\mathscr{E}_{M-r I}$ with respect to $\|\cdot\|_{T}$ is bounded as $T \downarrow 0$.

Lemma 3.2. If a constant $\delta>0$ satisfies

$$
\sigma(M) \subset\{\lambda \in \mathbb{C} ; \quad \operatorname{Re} \lambda<-\delta\}
$$

then there exists a constant $C>0$ such that

$$
\left\|\mathscr{E}_{M-r I} g\right\|_{T} \leq \frac{C}{r+\delta}\|g\|_{T} \quad \text { for } g \in C^{0}[0, T]
$$

where $C=C_{M, \delta}$ is independent of $T>0$ and $r \in \mathbb{N}_{0}$.

Proof. It suffices to choose $C$ in such a way that

$$
\left|s^{-M} v\right| \leq C s^{\delta}|v| \text { for } \mathrm{s} \in(0,1) \text { and } v \in \mathbb{C}^{N} \text {, }
$$

a fact which can be easily seen by writing $M$ in Jordan normal form. q.e.d.

\subsection{A nonlinear system; Proof of Theorem 2}

In order to complete the proof of Theorem 2, we consider a nonlinear system of singular ordinary differential equations

$$
t \frac{d u}{d t}(t)=f(t, u(t)) \quad \text { for } 0<t \leq T
$$

where $f, \partial f / \partial u \in C^{0}\left(\left[0, T_{0}\right] \times B\left(R_{0}\right)\right)$ with $0<T \leq T_{0}$ and $B\left(R_{0}\right)=\left\{v \in \mathbb{C}^{N}\right.$; $\left.|v| \leq R_{0}\right\}, R_{0}>0$. Assuming, as in (3.2), that

$$
M=\frac{\partial f}{\partial u}(0,0) \quad \text { satisfies }(3.4) \text { and } f(0,0)=0
$$

we shall prove the unique existence in the set $\mathscr{C}_{T, R}^{0.1}$ with $0<R \leq R_{0}$, where $\mathscr{C}_{T, R}^{r 11}=C^{r}([0, T], B(R)) \cap C^{r+1}(0, T]$, together with the corresponding regularity 
$u \in \mathscr{C}_{T, R}^{r, 1}$ for each $r \in N$ in case

$$
f, \frac{\partial f}{\partial u} \in C^{r}([0, T] \times B(R))
$$

More precisely,

Proposition 3. There exist constants $T \in\left(0, T_{0}\right]$ and $R \in\left(0, R_{0}\right]$ such that (3.7) has a unique solution $u$ in $\mathscr{C}_{T, R}^{0.1}$, which satisfies $u(0)=0$ automatically. Furthermore, $u \in \mathscr{C}_{T, R}^{r, 1}$ provided (3.9), holds with $r \in N$.

Proof of the unique existence. Setting $g[u](t)=f(t, u(t))-M u(t)$, we see that the equation (3.7) takes the form (3.3) with $g=g[u]$. It then follows from Lemma 3.1 that (3.7) with $u \in \mathscr{C}_{T . R}^{0,1}$ is equivalent to $u=\mathscr{F}[u]$, where $\mathscr{F}[u]=\mathscr{E}_{M} g[u]$. Thus, it suffices to show that

$$
\mathscr{F} \text { is a contraction on } \mathscr{C}_{T, R}^{0}=C^{0}([0, T], B(R))
$$

with respect to the norm $\|\cdot\|_{T}$ as far as the constants $T$ and $R$ are chosen appropriately.

We first observe that if $u_{1}, u_{2} \in \mathscr{C}_{T, R}^{0}$ then

$$
\mathscr{F}\left[u_{1}\right]-\mathscr{F}\left[u_{2}\right]=\mathscr{E}_{M} h,
$$

where $h(t)=\{\tilde{M}(t)-M\}\left\{u_{1}(t)-u_{2}(t)\right\}$ with

$$
\tilde{M}(t)=\int_{0}^{1} \frac{\partial f}{\partial u}\left(t, u_{2}(t)+\theta\left[u_{1}(t)-u_{2}(t)\right]\right) d \theta .
$$

It then follows from Lemma 3.2 that

$$
\left\|\mathscr{F}\left[u_{1}\right]-\mathscr{F}\left[u_{2}\right]\right\|_{T} \leq \frac{C}{\delta}\|h\|_{T} \leq L\left\|u_{1}-u_{2}\right\|_{T}
$$

with $L=(C / \delta) \max \{|\tilde{M}(t)-M| ; \quad 0 \leq t \leq T\}$, where $|\cdot|$ stands for the matrix norm. Clearly, $0 \leq L<1$ provided $T$ and $R$ are small enough. We next notice that if $u \in \mathscr{C}_{T, R}^{0}$ then

$$
\|\mathscr{F}[u]\|_{T} \leq L\|u\|_{T}+\|\mathscr{F}[0]\|_{T} \leq L R+\frac{C}{\delta}\|g[0]\|_{T},
$$


while $g[0](t)=f(t, 0)$, so that $\|\mathscr{F}[u]\|_{T} \leq R$ provided $T$ and $R$ are small enough. Therefore, the desired conclusion (3.10) is obtained under the assumption (3.8).

The proof above shows that $R \in\left(0, R_{0}\right]$ can be chosen arbitrarily small by shrinking $T \in\left(0, T_{0}\right]$. Hence, the unique solution $u \in \mathscr{C}_{T, R}^{0.1}$ must satisfy $u(0)=0$.

Proof of the regularity. It suffices to prove by induction on $r$ that

$$
u \in C^{r}[0, T] \text { provided }(3.9)_{r} \text { holds, }
$$

where $u \in \mathscr{C}_{T . R}^{0,1}$ is the unique solution of $(3.7)$, so that $(3.11)_{0}$ is valid. In fact, (3.11) r together with $u \in \mathscr{C}_{T, R}^{0,1}$ implies the desired conclusion $u \in \mathscr{C}_{T, R}^{r, 1}$. Recall that the unique existence was proved via the contraction principle, so that the unique solution $u=u_{\infty} \in \mathscr{C}_{T, R}^{0}$ (see (3.10)) is obtained by taking the limit in the iteration scheme

$$
u_{k+1}=\mathscr{F}\left[u_{k}\right]=\mathscr{E}_{M} g\left[u_{k}\right] \quad \text { for } \quad k \in \mathbb{N}_{0} ; \quad u_{0}=0
$$

It then follows from the differentiability property of $\mathscr{E}_{M}$ as in (3.6) that if (3.9) is assumed then $\left\{u_{k}\right\}_{k} \subset \mathscr{C}_{T, R}^{r, 1} \subset C^{r}[0, T]$ and

$$
u_{k+1}^{(r)}=\mathscr{E}_{M-r I} g\left[u_{k}\right]^{(r)} \quad \text { for } \quad k \in \mathbb{N}_{0} ; \quad u_{0}^{(r)}=0
$$

Thus, the desired conclusion $u_{\infty} \in C^{r}[0, T]$ will be obtained if we shall show that $\left\{u_{k}^{(r)}\right\}_{k}$ is uniformly convergent on $[0, T]$,

by shrinking $T>0$ if necessary.

Let us prove $(3.13)_{r+1}$ by assuming $(3.13)_{r}$ and $(3.9)_{r+1}$, so that $(3.12)_{r+1}$ is valid. Setting $w_{k}=u_{k}^{(r+1)}$, we regard $(3.12)_{r+1}$ as an iteration scheme for $\left\{w_{k}\right\}_{k}$ involving $u_{k}, u_{k}^{\prime}, \cdots, u_{k}^{(r)}$ as known functions. In order to write it down more explicitly, we begin by observing that $g\left[u_{k}\right]^{\prime}=A\left[u_{k}\right] u_{k}^{\prime}+b\left[u_{k}\right]$, where

$$
A[u](t)=\frac{\partial f}{\partial u}(t, u(t))-M, \quad b[u](t)=\frac{\partial f}{\partial t}(t, u(t)) .
$$

Differentiating both sides successively, we obtain $g\left[u_{k}\right]^{(r+1)}=A\left[u_{k}\right] w_{k}+b_{r}\left[u_{k}\right]$, 
where

$$
b_{r}[u]=\sum_{i=0}^{r-1}\left(\begin{array}{l}
r \\
i
\end{array}\right) \quad A[u]^{(r-i)} u^{(i+1)}+b[u]^{(r)}
$$

Then, $(3.12)_{r+1}$ can be written as

$$
w_{k+1}=\mathscr{A}_{r}\left[u_{k}\right] w_{k}+\mathscr{B}_{r}\left[u_{k}\right] \text { for } k \in N_{0} ; w_{0}=0,
$$

where $\mathscr{A}_{r}[u] w=\mathscr{E}_{M-(r+1) I}(A[u] w) \quad$ and $\quad \mathscr{B}_{r}[u]=\mathscr{E}_{M-(r+1) I} b_{r}[u]$.

The uniform convergence of $\left\{w_{k}\right\}_{k}$ is obtained via (3.14) as follows. We first observe by Lemma 3.2 that

$$
\left\|\mathscr{A}_{r}[u] w\right\|_{T} \leq \frac{C}{r+1+\delta}\|A[u] w\|_{T} \leq L^{\prime}\|w\|_{T}
$$

where $L^{\prime}=(1+\delta)^{-1} C \sup \left\{|A[u](t)| ; \quad 0 \leq t \leq T, u \in \mathscr{C}_{T, R}^{0}\right\}$ so that $0 \leq L^{\prime}<1$ provided $T$ and $R$ are small enough. Thus, there exists a unique $w_{\infty} \in C^{0}[0, T]$ satisfying

$$
w_{\infty}=\mathscr{A}_{r}\left[u_{\infty}\right] w_{\infty}+\mathscr{B}_{r}\left[u_{\infty}\right]
$$

Setting $\omega_{k}=w_{k}-w_{\infty}$, we have $\left\|\omega_{k+1}\right\|_{T} \leq L^{\prime}\left\|\omega_{k}\right\|_{T}+\varepsilon_{k}$ with $\varepsilon_{k} \downarrow 0$ as $k \rightarrow \infty$. This yields at first the boundedness of $\left\{\omega_{k}\right\}_{k}$, and then the convergence $\omega_{k} \rightarrow 0$ as $k \rightarrow \infty$, which implies the desired conclusion $(3.13)_{r+1}$. Therefore, the proof of Proposition 3 is complete.

We are now in a position to prove Theorem 2. By virtue of Propositions 2 and 3 , we can finish the proof with the aid of the formula $(3.6)_{0}$ as follows.

Proof of Theorem 2. It remains to show that the $C^{\infty}$ solution for each one of the one-sided problems continues smoothly across $t=0$ to that for the other one. But this fact is obvious if we write the equation (1.11) in the form (3.1). Indeed, the limits $u^{(r)}( \pm 0)$ can be computed successively by using the formula $(3.6)_{0}$, and it is easily verified that

$$
u^{(r)}(+0)=u^{(r)}(-0) \text { for } \quad r \in N_{0}
$$

Let us explain it more precisely. By virtue of Proposition 3, we see that the $C^{\infty}$ solution $v(t)=t u(t)$ of $(1.11)$ for $0 \leq t \leq T$ exists and that the 
solution $u(t)$ is unique in the class $\mathscr{C}_{T}^{0,1}=C^{0}[0, T] \cap C^{1}(0, T]$, where $T>0$ is small enough. The same is true for the problem on $[-T, 0]$. Indeed, setting $u_{ \pm}(t)=u( \pm t)$, we are led to the equations

$$
t \frac{d}{d t} u_{ \pm}(t)=f_{ \pm}\left(t, u_{ \pm}(t)\right) \quad \text { for } \quad 0<t \leq T
$$

where $f_{ \pm}(t, u)=f( \pm t, u)$, so that $f_{-}(0,0)=f_{+}(0,0)=0$ and

$$
\frac{\partial f_{-}}{\partial u}(0,0)=\frac{\partial f_{+}}{\partial u}(0,0)=M
$$

Therefore, Proposition 3 can be applied equally to both problems. It remains to verify (3.15), i.e.

$$
u_{-}^{(r)}(+0)=(-1)^{r} u_{+}^{(r)}(+0) \text { for } r \in \mathbb{N}_{0}
$$

Setting $g_{ \pm}(t)=f_{ \pm}\left(t, u_{ \pm}(t)\right)-M u_{ \pm}(t)$, we have

$$
\frac{d}{d t} u_{ \pm}(t)-M u_{ \pm}(t)=g_{ \pm}(t)
$$

It then follows from the formula $(3.6)_{0}$ that

$$
u_{ \pm}^{(r)}(+0)=(r I-M)^{-1} g_{ \pm}^{(r)}(+0)
$$

so that $(3.15)^{\prime}$ is equivalent to

$$
g_{-}^{(r)}(+0)=(-1)^{r} g_{+}^{(r)}(+0) \text { for } \quad r \in \mathbb{N}_{0}
$$

Recall $u_{ \pm}(+0)=0$, so that $g_{ \pm}(+0)=0$. On the other hand, it is clear from the definition of $g_{ \pm}(t)$ that $g_{ \pm}^{(r)}(+0)$ are independent of $u_{ \pm}^{(r)}(+0)$ and depend only on $u_{ \pm}^{\left(r^{\prime}\right)}(+0)$ with $r^{\prime}<r$. Therefore, the desired conclusion $(3.15)^{\prime \prime}$ is obtained by induction on $r$.

\section{References}

[1] Briot, C. et Bouquet, J.C., Recherches sur les propriétés des fonctions définies par des équations différentielles, J. École Imp. Polytech. (1), cah. 36 (t. 21) (1856), 133-198.

[2] Hille, E., Ordinary Differential Equations in the Complex Domain, Wiley-Interscience, 
New York, 1976.

[ 3 ] De Hoog, F.R. and Weiss, R., Difference methods for boundary value problems with a singularity of the first kind, SIAM J. Numer. Anal., 13 (1976), 775-813.

[ 4 ] - On the boundary value problem for systems of ordinary differential equations with a singularity of the second kind, SIAM J. Math. Anal., 11 (1980), 41-60.

[5] Hörmander, L., The Cauchy problem for differential equations with double characteristics, J. Analyse Math., 32 (1977), 118-196.

[6] - The Analysis of Linear Partial Differential Operators III, Grundlehren Math. Wiss., 274, Springer,, Berlin, 1985.

[ 7 ] Hukuhara, M., Kimura, T. et Matuda, T., Équations Différentielles Ordinaires du Premier Ordre dans le Champ Complexe, Publ. Math. Soc. Japan, 7, Math. Soc. Japan, Tokyo, 1961.

[ 8 ] Ince, E.L., Ordinary Differential Equations, Longmans, Green and Co. Ltd., London, 1927.

[9] Ivrii, V. Ya. and Petkov, V.M., Necessary conditions for the Cauchy problem for non-strictly hyperbolic equations to be well-posed, Uspekhi Mat. Nauk, 29:5 (1974), 3-70; English translation in Russian Math. Surveys, 29:5 (1974), 1-70.

[10] Iwasaki, N., The Cauchy problem for effectively hyperbolic equations (a standard type), Publ. RIMS, Kyoto Univ., 20 (1984), 543-584.

[11] - The Cauchy problem for effectively hyperbolic equations (Remarks), in Hyperbolic Equations and Related Topics (Proc. Taniguchi Intern. Symp., Katata and Kyoto, 1984), ed. by S. Mizohata, pp. 89-100, Kinokuniya, Tokyo, 1986.

[12] Melrose, R., The Cauchy problem for effectively hyperbolic operators, Hokkaido Math. J., 12 (1983), 371-391.

[13] Nishitani, T., On wave front sets of solutions for effectively hyperbolic operators, Sci. Rep. College Gen. Ed. Osaka Univ., 32:2 (1983), 1-7.

[14] Russell, D.L., Numerical solution of singular initial value problems, SIAM J. Numer. Anal., 7 (1970), 399-417. 
